\title{
A Mild Case of Adult-Onset Keratosis Lichenoides Chronica Successfully Treated with Narrow-Band UVB Monotherapy
}

\author{
Takashi Nomura $^{\mathrm{a}}$ Eiko Toichi $^{\mathrm{b}}$ Yoshiki Miyachi $^{\mathrm{a}}$ \\ Kenji Kabashima ${ }^{a}$ \\ Departments of Dermatology at ${ }^{a}$ Kyoto University Graduate School of \\ Medicine, Kyoto University and ${ }^{b}$ Kyoto Medical Centre, Kyoto, Japan
}

\section{Key Words}

Keratosis lichenoides chronica - Narrow band-ultraviolet B - Monotherapy

\begin{abstract}
Keratosis lichenoides chronica $(K L C)$ is a rare keratotic disorder of unknown origin. Effective treatment has not been established yet. Here, we present adult-onset KLC, which was treated by narrow-band ultraviolet B (NB-UVB) monotherapy. Although NB-UVB was reported to be effective for pediatric-onset KLC, it has not been clear if this regimen is also useful for adult-onset type because pediatric-onset KLC is suggested to be different in nature. We assume that KLC is treatable by NB-UVB regardless of the age of the patient.
\end{abstract}

\section{Introduction}

Linear and reticulate keratotic lesions characterize keratosis lichenoides chronica (KLC). Kaposi described this rare disease in 1895 and Margolis coined the name KLC in 1972 [1]. Treatment of KLC is difficult and not established. Herein, we present an adult patient who was successfully treated with narrow-band ultraviolet B (NB-UVB) monotherapy.

\section{Case Report}

A 34-year-old female noticed stinging erythematous rashes on her trunk, which disappeared spontaneously within 3 days. A few days later, she found another array of rashes on her extremities. 
Older rashes disappeared, leaving brown pigmentation, while new ones continued to emerge. She eventually had arrays of annular erythematous psoriatic plaques that coalesced to form livedo on her extremities when she presented 1 month later (fig. 1a). She did not show nail changes or palmoplantar keratoderma. Topical salves such as dexamethasone propionate; maxacalcitol, a vitamin D3 derivative; and tacrolimus were not effective. Complete blood counts and laboratory findings were normal. The patient was negative for SSA, SSB, anti-nuclear antibody, anti-double stranded DNA, and a lupus band test.

Examination of biopsied skin from the leg revealed parakeratosis, follicular plugging, a slightly thickened granular layer, slight lymphocyte-infiltration at the epidermal-dermal junction with limited liquefaction, and mild perivascular lymphocyte-infiltration (fig. 2). These findings were consistent with KLC.

The differential diagnoses of KLC include lichen planus (LP), discoid lupus erythematosus (DLE), lichen planopilaris, pityriasis rubra pilaris (PRP), acquired ichthyosis, pityriasis lichenoides chronica (PLC), drug eruption, and mycosis fungoides (MF) including parapsoriasis. Reticular pattern of lesions, sparse lymphocyte-infiltration, and parakeratosis were not typical of LP. No indicative findings such as vesicles or scarring alopecia of DLE on sun-exposed areas, scarring alopecia of lichen planopilaris, or characteristic erythema of PRP were identified. Acquired ichthyosis was unlikely because the patient was free from malignancy, malnutrition, hypothyroidism, or diabetes mellitus. Eruptions along Blaschko's lines were not typical of PLC. Her medical history excluded drug eruption and ruled out MF or parapsoriasis. Thus, a mild case of KLC was the most likely diagnosis. Although our case lacked seborrheic dermatitis-like lesions, which Boer [1] found in typical KLC, the mildness of our case may have spared the facial lesions.

Because initial treatment with corticosteroids, a vitamin D3 derivative, and tacrolimus failed, the patient was treated with NB-UVB every other week. The lesions were very much improved after 3 months of treatment, when the total dosage reached $6.80 \mathrm{~J} / \mathrm{cm}^{2}$ (fig. $1 \mathrm{~b}$ ). The patient is under observation and thus far there are no signs of recurrence or findings suggestive of parapsoriasis.

\section{Discussion}

KLC is resistant to topical and systemic steroids, sulfones, methotrexate, antimalarial agents, radiation, and cyclosporine [1-4]. Acitretin, isotretinoin, etretinate, psoralen with UVA (PUVA), retinoid-PUVA, and topical calcipotriol have been used with various efficacies [3]. Recently, topical tacrolimus was reported to be effective in juvenile-onset KLC [5], although ineffective in our case, possibly reflecting a difference in the epidermal absorption rate. NB-UVB was also effective in treating pediatric-onset KLC in siblings (a 10-year-old girl and her 1.5-year-old brother) [6]. Although it is not clear whether pediatric-onset KLC is a subset of adult-onset KLC or a different disease entity [7], our patient responded well to NB-UVB.

To summarize, a mild case of adult-onset KLC was successfully treated with NB-UVB monotherapy. We propose that NB-UVB should be considered in treating KLC regardless of the patient's age.

\section{Disclosure Statement}

This work was supported in part by Grants-in-Aid for Scientific Research from the Ministries of Education, Culture, Sports, Science and Technology, and Health Labor, and Welfare of Japan. 

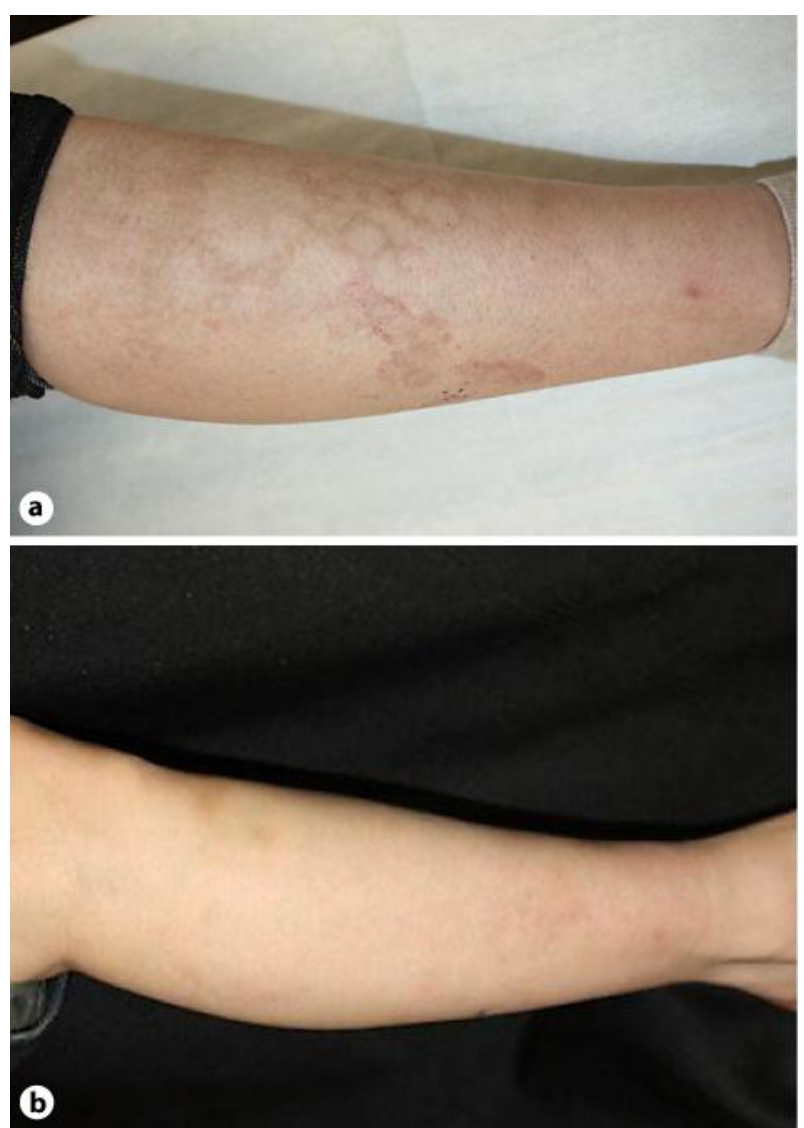

Fig. 1. KLC: clinical features of the eruption before (a) and after (b) NB-UVB monotherapy. a Array of annular erythematous psoriatic plaques on the right leg before treatment. Each plaque coalesced to form a livedo-like appearance. Older lesions left brown pigmentation while new ones appeared with a scarlet color. Black dots on the calf indicate the site of biopsy. b Most of the psoriatic plaques disappeared after the series of NB-UVB treatments. 


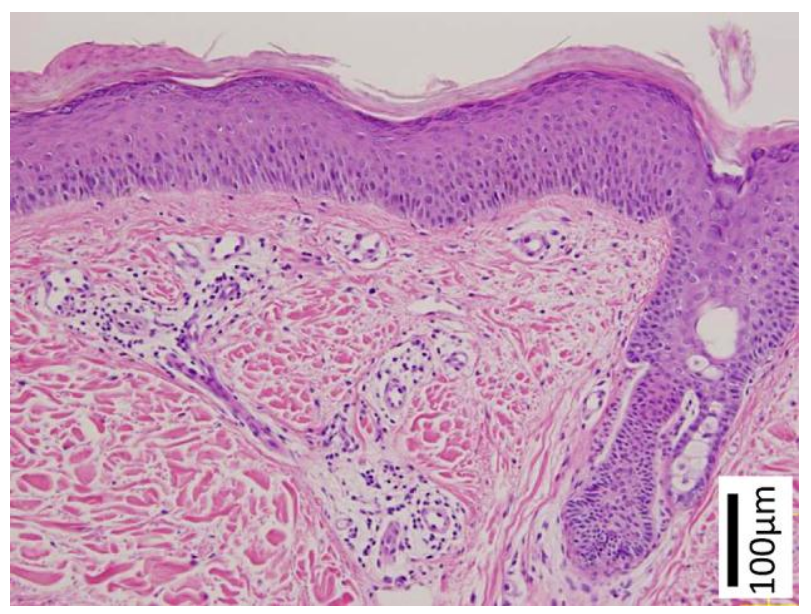

Fig. 2. KLC: histology of the biopsied skin, formalin-fixed and stained with hematoxylin-eosin staining. Epidermis showed parakeratosis, a mildly thickened granular layer, and follicular plugging. Dermal blood vessels were dilated and accompanied by mild perivascular lymphocyte infiltration. Scale bar $=100 \mu \mathrm{m}$.

\section{References}

1 Boer A: Keratosis lichenoides chronica: proposal of a concept: Am J Dermatopathol 2006;28:260-275.

$\checkmark 2$ Demirci E, Boyvat A, Arica IE, Kocyigit P, Ozdemir E, Heper AO: Keratosis lichenoides chronica: marked response to PUVA in combination with acitretin. Acta Derm Venereol 2006;85:552-553.

-3 Koseoglu RD, Sezer E, Yuksek J: Keratosis lichenoides chronica treated with acitretin plus narrowband ultraviolet B phototherapy. J Dermatol 2008;35:172-174.

4 Munoz-Santos C, Yebenes M, Romani J, Luelmo J: Response of keratosis lichenoides chronica to efalizumab therapy. Arch Dermatol 2009;145:867-869.

-5 Oyama N, Mitsuhashi Y, Yamamoto T: Juvenile-onset keratosis lichenoides chronica treated successfully with topical tacrolimus: a safe and favourable outcome. Eur J Dermatol 2011;21:595-596.

6 Tomb R, Soutou B: Keratosis lichenoides chronica in two siblings with marked response to UVB phototherapy (in French). Ann Dermatol Venereol 2008;135:835-838.

7 Ruiz-Maldonado R, Duran-McKinster C, Orozco-Covarrubias L, Saez-de-Ocariz M, Palacios-Lopez C: Keratosis lichenoides chronica in pediatric patients: a different disease? J Am Acad Dermatol 2007;56:S1-S5. 